\title{
Influence of parameters during induction heating cycle of 7075 aluminium alloys with RAP process
}

\author{
G. Vaneetveld ${ }^{1,}$, A. Rassili ${ }^{1, b}$ and H. V. Atkinson ${ }^{2, c}$ \\ ${ }^{1}$ ThixoUnit Ulg, PiMW B56, University of Liège, Sart Tilman, 4000 Liège, Belgium \\ ${ }^{2}$ Department of Engineering, University of Leicester, University Road, Leicester LE1 7RH, UK \\ ${ }^{a}$ G.Vaneetveld@ulg.ac.be, ${ }^{b}$ A.Rassili@ulg.ac.be, ${ }^{c}$ hva2@leicester.ac.uk
}

Keywords: thixoforging, 7075 alloys, hot tool, solid fraction, aluminium, semi-solid.

\begin{abstract}
Thixoforging involves shaping alloys with a globular microstructure in the semi-solid state. To reach this kind of material, the Recrystallisation and Partial Melting (RAP) process can be used to obtain a globular microstructure from extruded material with liquid penetrating the recrystallised boundaries. Induction heating is used to apply the RAP process to slugs. One of the benefits of using this method of heating is the fast heating rate $\left(20^{\circ} \mathrm{C} / \mathrm{s}\right)$. This paper will help to improve heating parameters by showing their influence on 7075 aluminium alloy recrystallisation. These parameters are the heating rate; heating frequencies-power; presence or not of protective gas; position of the slug in the inductor; energy stored inside the slug; oxide layer on the slug side; chamfer of the slug upper corner.
\end{abstract}

\section{Introduction}

Thixoforging process. Thixoforming is a semisolid metal processing route (SSM), which involves forming of alloys in the semisolid state to near net shaped products [1]. For this to be possible, the microstructure of the semisolid must consist of globular solid grains surrounded by a liquid matrix. It will then behave thixotropically: if it is sheared, the viscosity falls and it flows like a liquid, but if allowed to stand it thickens again. Thixoforging is thixoforming process at high solid fraction. With such a process, parts are shaped in one step and their mechanical properties are near those from forging process with complex geometry possibilities [2].

Material. The required microstructure can be obtained by several routes. In the present work, alloy $7075(\mathrm{Al}-(5.1-6.1) \mathrm{Zn}-(2.1-2.9) \mathrm{Mg}-(1.2-2.0) \mathrm{Cu}-0.5 \mathrm{Fe}-0.4 \mathrm{Si}-(0.18-0.28) \mathrm{Cr}-0.3 \mathrm{Mn}-$ $0.2 \mathrm{Ti}$, all composition in wt-\% unless otherwise stated [3]) supplied by ??? is studied. The 7075 aluminium alloy is a wrought alloy that shows, by DSC analysis, a less sensitive solid fraction to temperature variation at high solid fraction (Fig. 1); this is why we are going to focus here on a solid state route to obtain small solid globular grains surrounded by a liquid matrix. This solid state route is the recrystallisation and partial melting route (RAP) [4].

Firstly, the material is warm worked e.g. by extrusion. The extrusion has taken place below the recrystallisation temperature and the starting material for this work has the long, thin grains, typical of an extruded microstructure. Secondly, on reheating into the semisolid state, recrystallisation occurs and the liquid penetrates the recrystallised boundaries so resulting in spheroids surrounded by liquid. There are two main advantages of this solid state route. Firstly,

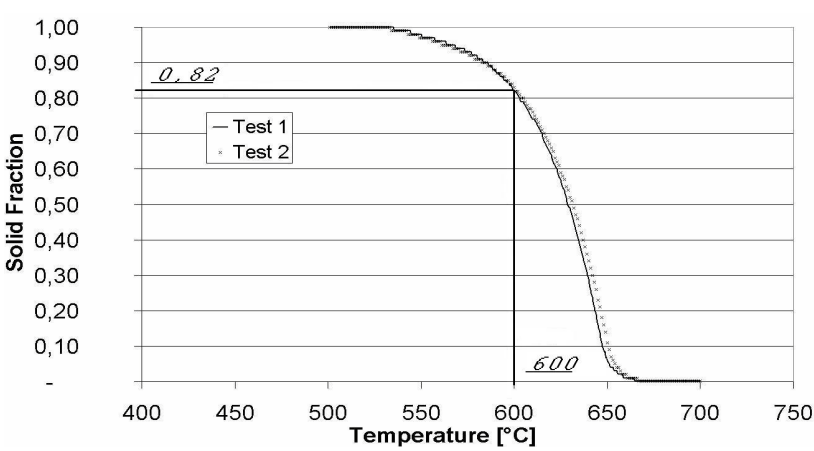

Fig. 1: Solid fraction vs. temperature obtained by DSC analysis at a heating rate of $15^{\circ} \mathrm{C} / \mathrm{min}$ some alloys are supplied in the extruded state (i.e. worked) in any case. Secondly, the spheroids are 
more fully rounded than those from, for example, the standard commercial route to spheroidal microstructures, like magnetohydrodynamic (MHD) stirring. The main disadvantages are that there may be variation in the amount of stored work across the section, resulting in inhomogeneity, and extrusion can be difficult and expensive with wider slug ${ }^{1}$ diameters [5]. In the RAP process, some heating parameters have to be optimised to reduce time for industrial application while keeping in mind the quality of parts. Effects of these parameter variations on the microstructure are observed.

\section{Experiences}

Inductive heating is used to apply the RAP process to a slug placed on a ceramic pedestal at room temperature. The seven heating parameters we focus on are: heating rate; heating frequency-power; position of the slug in the inductor; the presence, or not, of protective gas; energy stored in the slug; oxide layer on the side of the slug and chamfer. Their effects on the recrystallisation are described. To check these influences, a $150 \mathrm{~kW}$ generator with frequencies from $2 \mathrm{kHz}$ to $10 \mathrm{kHz}$ is used with a paraboloic inductor, shown in figure 2 , to reach homogeneous temperature from the bottom to the top of the slug during heating.

Heating rate. Heating cycle length is an important parameter for industrial application. In this goal, two heating cycles with different lengths, reaching $600^{\circ} \mathrm{C}$, are compared from the point of view of different energies and recrystallisation. These heating cycles reach the final temperature relatively slowly to let kinetic transformations, like solid to liquid, occur. Microstructures of slugs heated with these two heating cycles are compared. For the next set of experiments, a short

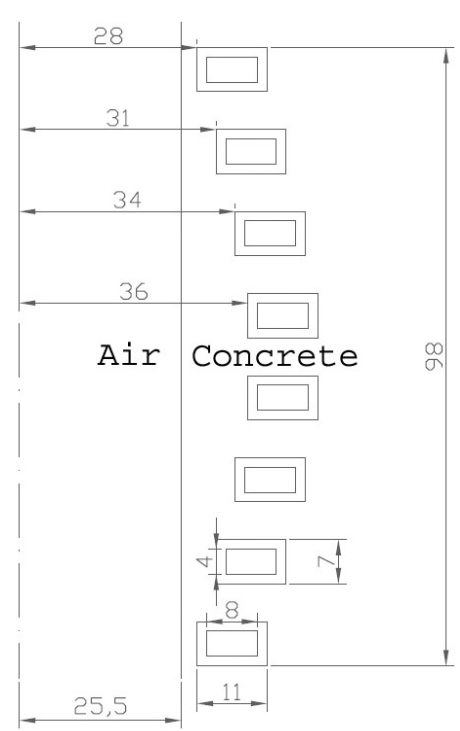

Fig. 2 : Inductor dimensions in $[\mathrm{mm}]$ improved heating cycle is then used.

Heating frequency/power. Two heating cycles, having different frequencies and showing the same evolution of temperature by modifying the power, are tested. The frequencies tested are the lowest one on our $150 \mathrm{~kW}$ generator, $2,3 \mathrm{kHz}$, and a higher one using maximal power for the first step: $5,4 \mathrm{kHz}$. For the slug, using different frequencies means different penetrations. Recrystallisation is studied for each heating cycle. For this, three samples from each heating cycle at different frequencies are cut to observe microstructure. For the other experiments, low frequency is used.

Position. It's quite hard to build an inductor where the centre corresponds to the electomagnetic field axis (EMFA). So on our device, the EMFA is not in the centre of the inductor, and the power is so high at the beginning of the heating cycle that, due to the light weight of aluminium, the slug moves against the cement that protects the coils of the inductor. There is an energy loss by conduction that could affect the recrystallisation of the slug. Three slugs $(30 \mathrm{~mm}$ diameter, $59 \mathrm{~mm}$ length) not centred in the inductor are heated in the same condition. Slugs are cut in quarters: microstructure in contact with the cement is observed. The side of the slugs is examined to note any differences in recrystallisation.

Protective gas. To observe the influence of protective gas on the recrystallisation at the side surface of the slug, different pressures of argon are tested: 3 bars, 1 bar and no argon ( 0 bar i.e. air). The heating cycle has to be modified to compensate the loss by convection due to the gas. The side of the slugs is observed to note any differences in recrystallisation.

Energy stored. During rolling of the feedstock, friction on the side of the extruded bar creates heat that could decrease energy stored in these areas and affect recrystallisation during heating to

\footnotetext{
${ }^{1}$ Small cylinder cut from extruded bar.
} 
semisolid state [5]. To avoid influence of other parameters, the oxide layer on extruded bar is removed by machining $0,2 \mathrm{~mm}$, no argon is used and the position of the slug during heating is in the centre of the inductor. A slug of $59 \mathrm{~mm}$ length is cut from a $35 \mathrm{~mm}$ diameter 7075 extruded bar. The side of the slugs is examined to note any differences in recrystallisation.

Chamfer. Influence of chamfer on the slug upper corner, in relation to homogeneity of the solid fraction in this area, is studied. A slug with chamfer and another without are heated in the same conditions. The solid fraction homogeneity is observed on the microstructure of the slug in the upper corner.

\section{Results and Discussion}

Heating rate. Two different heating cycle lengths and the corresponding powers, reaching $600^{\circ} \mathrm{C}$, are shown on figure 3 . It is difficult to reach smoothly the final temperature because our generator can only give steps in levels of power (Fig. 4). The sensibility of the power applied on the slug is about $0,1 \mathrm{~kW}$.

The comparison between microstructures from each cycle is shown in figure 5. Grain size, shape and solid fraction are the same for both cycles. But some non recrystallized grains remain for

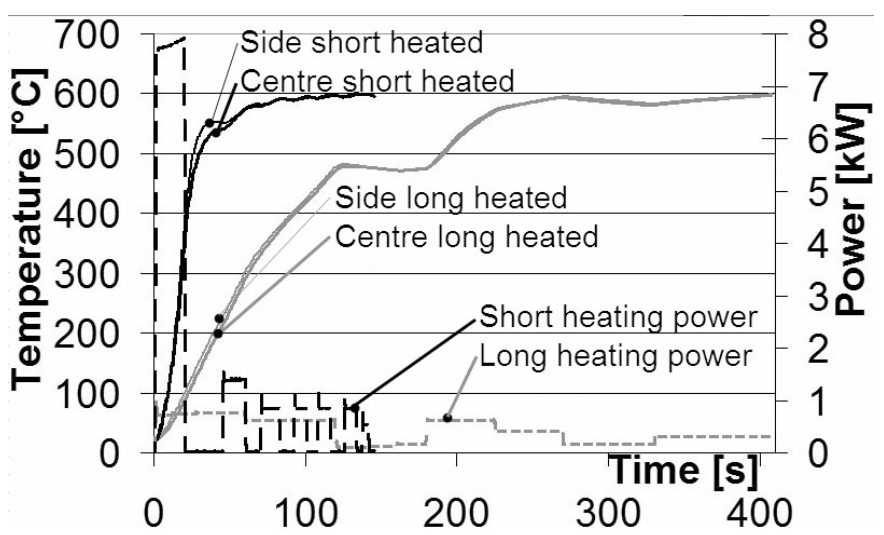

Fig. 3: Different heating cycles lengths with corresponding power cycles (Need to explain more clearly)

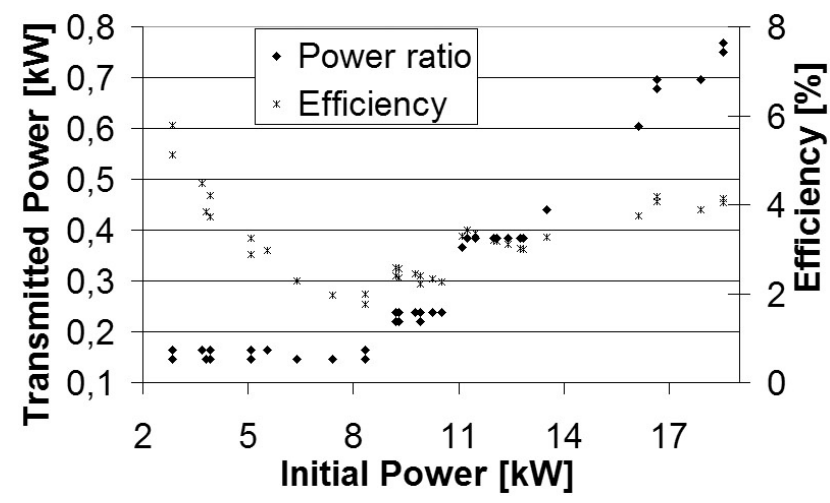

short cycle on the Fig. 4: Power levels given by our generator (Can you give a larger point size so the diagram is clearer??? axis of the slug due to the low energy stored in the axis during extrusion. We will observe influence of these elongated grains on thixoforged parts [6].

From an energy point of view, a short heating cycle of $145 \mathrm{~s}$ consumes $8 \%$ more than the longer one at $415 \mathrm{~s}$ in reaching the same temperature (Fig. 3) because of the power transmission saturation to the slug.

If a protective atmosphere is used during heating, the possibility of reducing heating cycle length is interesting from a cost point of view.

Heating frequency/power. Two heating cycles at different frequencies $(2,3 \mathrm{kHz}$ and $5,4 \mathrm{kHz})$ showing the same evolution of temperature (Fig. 6)

by modifying heating power are tested.

It is difficult to have the same temperature evolution at two different frequencies because of the

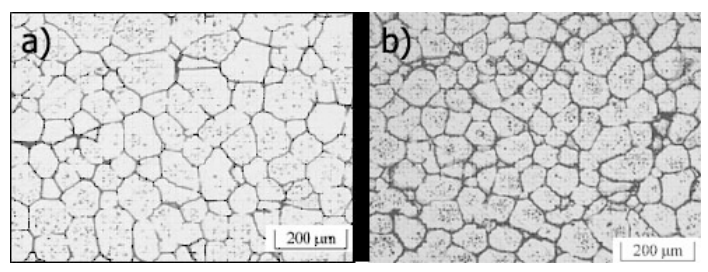

Fig. 5: Microstructures etched in Keller reagent of slugs heated with cycle from figure 3: a) short cycle; b) long cycle

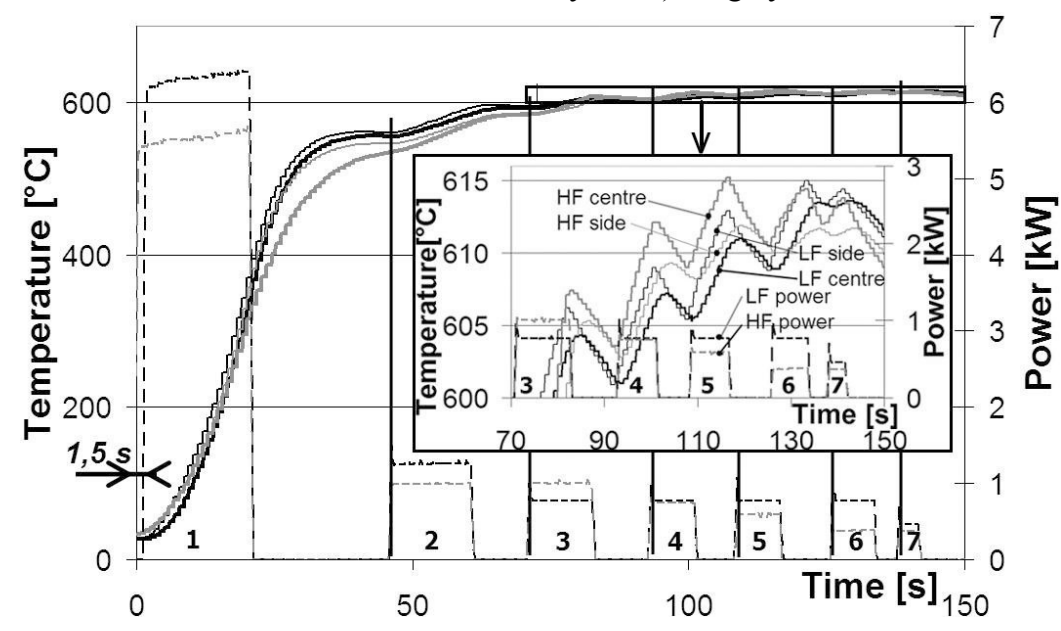

Fig. 6: Temperature evolution from two heating cycles having different frequencies using different powers 
difference in power efficiency between low frequency (LF) and high frequency (HF). First, we notice on figure 6 that the power at LF starts with a delay of 1,5 second (this isn't clear in the diagram....). On the same figure, the temperature in the slug is more homogeneous during heating for LF than for HF. The HF overall power used to heat the slug, is lower than the LF one. For the first power step, the LF power, where the efficiency on our device is $8 \%$ is twice as efficient as for the HF power (4\%) because of the power transmission saturation to the slug. So it is advantageous for homogeneous temperature and cost saving to use LF.

To calculate the penetration for different frequencies, the next formula is used [7]: $\mathrm{p}^{2}=\rho /\left(\pi^{*} \mu_{0} * \mu_{\mathrm{r}} * \mathrm{f}\right)$ where $\mathrm{p}$ is the penetration in $[\mathrm{m}]$, $\rho$ is the electric resistivity in $\left[\Omega^{*} \mathrm{~m}\right], \mu_{0}$ is the

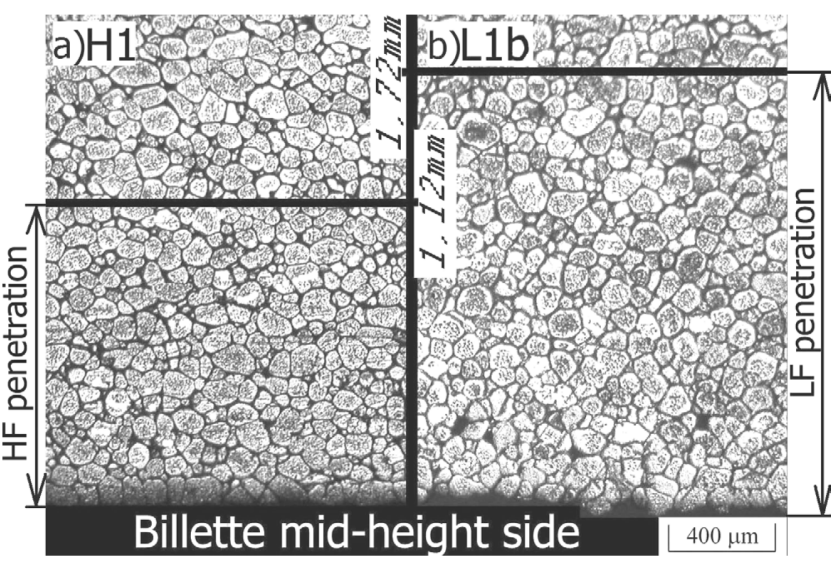

Fig. 7: Microstructures etched in Keller reagent from slugs heated at two different frequencies with cycles shown on figure 6 vacuum permeability in $[\mathrm{H} / \mathrm{m}], \mu_{\mathrm{r}}$ is the relative permeability, $\mathrm{f}$ is the frequency in $[\mathrm{Hz}]$ :

- $\mathrm{p}_{\mathrm{LF}}=1,72 \mathrm{~mm}$ for LF heating;

- $\mathrm{p}_{\mathrm{HF}}=1,12 \mathrm{~mm}$ for HF heating.

The difference between penetration distance for $\mathrm{LF}$ and $\mathrm{HF}$ is $0,6 \mathrm{~mm}$.

The comparison between microstructures of slug side from each cycle is shown on figure 7. All

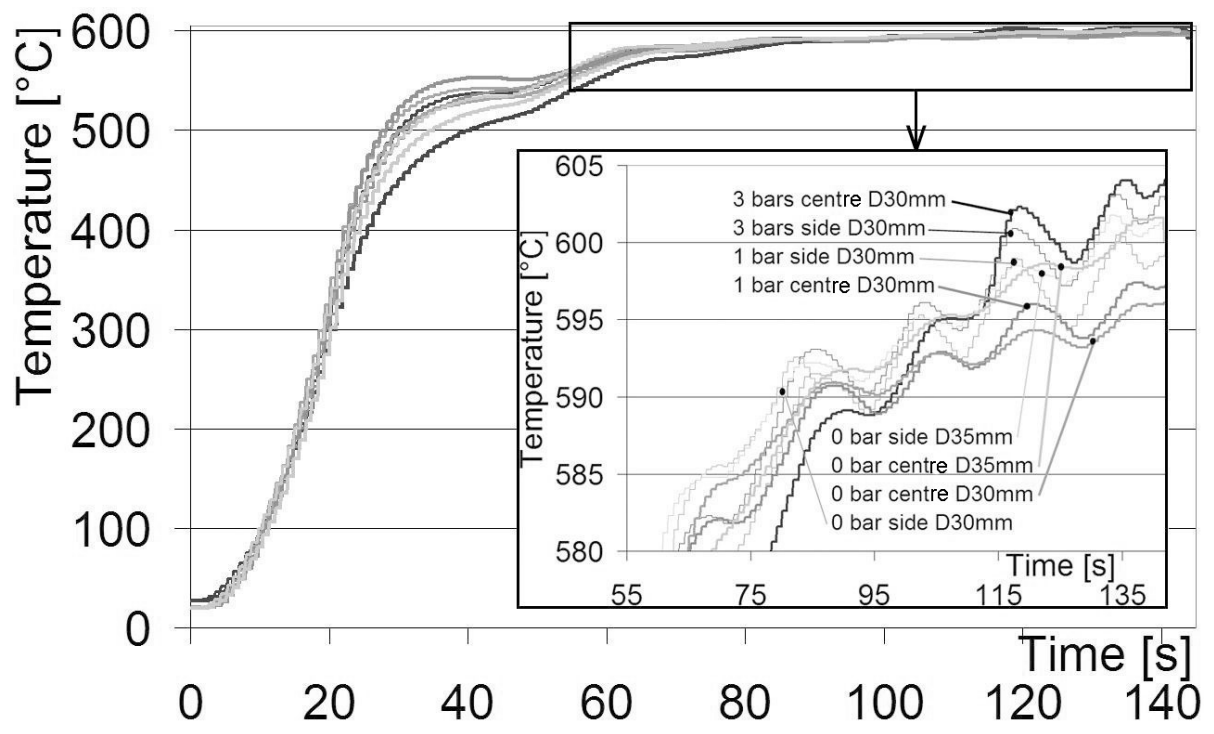
grains have the same size and shape. There is no transition around the limit of the penetration distance from recrystallised grains point of view because of the conduction. There is no difference in recrystallisation on the slug side between grains heated at $\mathrm{HF}$ and

Fig. 8: Heating cycles used for different pressures of argon: 3,1 or 0 bars for two diameters: D30 $\mathrm{mm}$ or D35mm those heated at LF because they are heated at the same power (Fig. 6) but on different thickness.

Position. There is no difference about microstructures from recrystallisation point of view. If the slug touches the cement of the inductor, the contact is so small that little heat

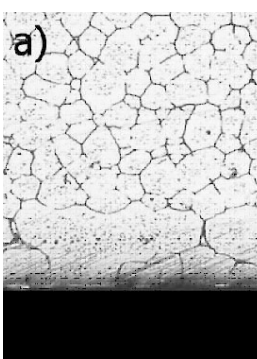

Fig. 9: Microstructures etched in Keller reagent from slugs D30mm heated with cycles shown on figure 8 at different argon pressures : a) 3 bars, b) 1 bar, c) no argon (0 bar) is lost to modify the recrystallisation. Ensuring the inductor diameter is matched to the slug diameter:

- increases efficiency and homogeneity of the temperature in the slug during heating; 
- limits the displacement of the slug during heating at high power.

Protective gas. The convection between the argon and the slug means that the heating power cycle has to be modified to achieve the same temperature evolution (Fig. 8).

The comparison of microstructures shows, in figure 9, that there is a zone of large grains at the side of the specimen for higher argon pressure and this hardly exists for 0 bar (no argon). These large grains have the same composition as globular grains inside the slug (Table microstructure (although a very thin layer may be present).

\begin{tabular}{|c|c|c|c|c|}
\hline in [Wt\%] & $\mathrm{Al}$ & $\mathrm{Zn}$ & $\mathrm{Mg}$ & $\mathrm{Cu}$ \\
\hline $\begin{array}{c}\text { Large surface } \\
\text { grains }\end{array}$ & 69,9 & 16,0 & 9,3 & 4,9 \\
\hline $\begin{array}{c}\text { Recrystallised } \\
\text { grains in the } \\
\text { centre }\end{array}$ & 69,0 & 16,3 & 9,3 & 5,3 \\
\hline
\end{tabular}

Table 1: Chemical composition comparison between large surface grains and those in the centre from EDX analysis

1). No oxide layer is observed on A benefit of gas is that it assists in rapidly achieving a homogeneous temperature distribution between the centre and the side of the slug. Another positive point is that, at high temperature (over $615^{\circ} \mathrm{C}$ ), the gas helps to maintain the shape of the slug, avoiding runoff due to gravity.

Energy stored. Figure 10 shows the transition between the machined side length where the oxide layer has been removed and the non-machined region (***Need to label on the micrograph). There is little difference between the two regions. There is however a layer about $1 \mathrm{~mm}$ in from the surface where some elongated grains are still present. These grains might interfere with flow in thixoforming. If this was found to be the case, this could be avoided by machining a layer $2 \mathrm{~mm}$ thick from the surface.

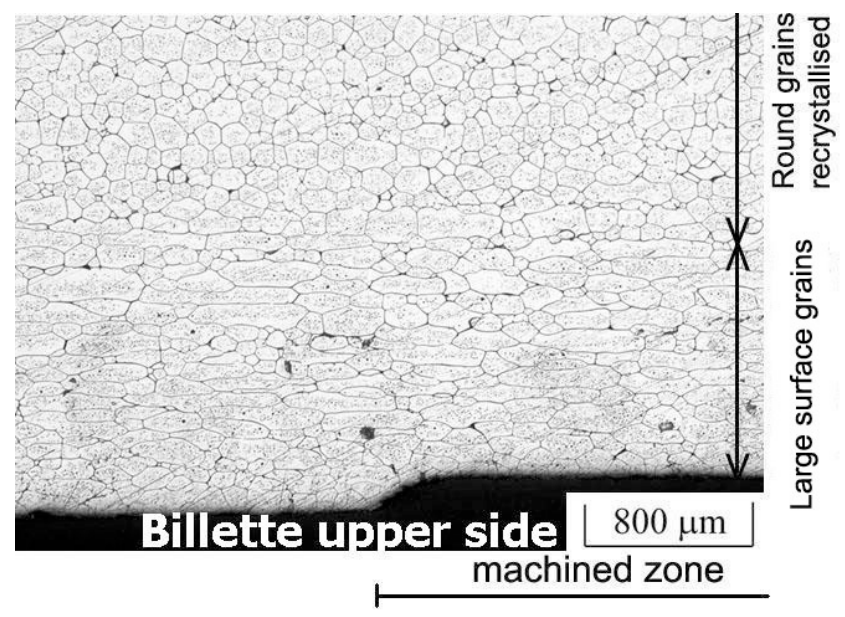

Fig. 10: Microstructure etched in Keller reagent of the transition between machined zone and the nonmachined zone from a slug heated with cycle 0 bar D35mm shown on figure 8

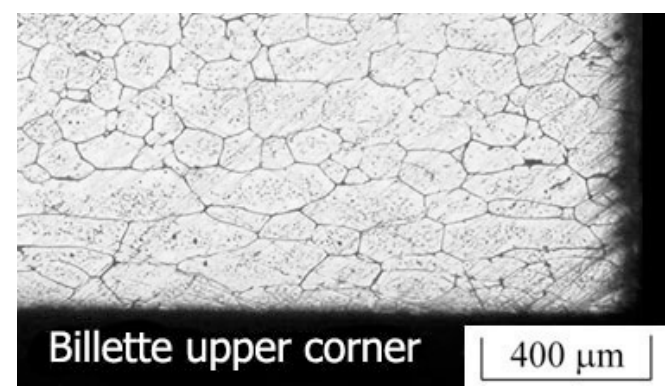

Fig. 11: Microstructure etched in Keller reagent of the upper corner from a slug heated with cycle 0 bar D $35 \mathrm{~mm}$ shown on figure 8

Chamfer. No solid fraction variation is observed in the upper side corner due to the right angle (Fig. 11). It is not therefore necessary to machine a chamfer on the top corner of the slugs (as is argued by some researchers) for the temperatures used here $\left(600^{\circ} \mathrm{C}\right)$.

\section{Conclusions}

In the RAP process, some parameters have to be optimised to reduce time for heating into the semi-solid state while keeping in mind the quality of parts. Influences of heating parameters on recrystallisation have been observed. To observe these influences, we had to apply the same temperature evolution to slugs in different conditions sometimes by modifying power levels:

- Heating cycle length is reduced to $145 \mathrm{~s}$ by increasing heating rate to $20^{\circ} \mathrm{C} / \mathrm{s}$ for the first power step. There are no major impacts on the microstructure (grain size, shape or solid fraction). A few unrecrystallised grain remain but their resistance to the material flow 
during thixoforging should be minor due to their low thickness [6]. A seven step heating cycle is used to approach slowly, in a homogeneous way, a target temperature whilst allowing kinetic transformations like the solid-liquid transition to occur.

- Low frequency heating at $2,3 \mathrm{kHz}$ is twice as efficient on our device as 5,4 kHz. The frequency variation does not significantly influence recrystallisation.

- The position of the slug in the inductor does not have an influence on the recrystallisation.

- The use of a protective gas leads to large grains on the side of the slug.

- Another source of low recrystallised grains is the side of the extruded material. In this zone, there is not enough stored energy because of friction during rolling to reach round recrystallised grains. This layer, about 2 to $3 \mathrm{~mm}$ on diameter, should be machined to have a microstructure with full round solid grains. There is no influence observed of oxide layer on recrystallisation.

- The chamfer on the top of slugs is not needed at $600^{\circ} \mathrm{C}$ because there is no variation of solid fraction in the upper corner of the slug.

\section{Acknowledgement}

The authors gratefully acknowledge University of Liège and Walloon Region (First Europe Project) for their support. We would like to thanks J.-C. Pierret for the hand to heat samples, G. Clarke for the training on the EDX and S. Salieri for the help in the sample preparation.

\section{References}

[1] M. C. Flemings: Metall. Trans. A, 1991, 22A, 957-981.

[2] G. Vaneetveld, A. Rassili, J. Lecomte-Beckers, H.V. Atkinson; Thixoforging of 7075 aluminium alloys at high solid fraction; Trans. Tech Publications, Solid State Phenomena Vols. 116-117, p. 762-765, Switzerland, 2006.

[3] Metals Handbook ${ }^{\circledR}$, Desk Ed., ASM, Ohio, 1984.

[4] D.H. Kirkwood, C.M. Sellars, L.G. Elias-Boyed. Thixotropic materials. European Patent No. 0305375 B1; 28 October 1992.

[5] K. Burke, G. Vaneetveld, H.V. Atkinson: Recrystallisation in the semi-solid state in 7075 Aluminium Alloy, for publication in Material Sciences and Engineering.

[6] G. Vaneetveld, A. Rassili, H. V. Atkinson; Improvement in thixoforging of 7075 aluminium alloys at high solid fraction; submitted to S2P Conference 2008.

[7] M. Suéry; Mise en forme d'alliages métalliques à l'état semisolide; Mécanique et Ingénierie des matériaux, Lavoidier-Hermes, 2002. 